\title{
Review
}

\section{Evolving Frontline Treatment Landscape for Advanced or Metastatic Renal Cell Carcinoma}

\author{
Rohit K. Jain $^{\mathrm{a}, *}$ and Primo N. Lara ${ }^{\mathrm{b}}$ \\ ${ }^{a}$ Department of Genitourinary Oncology, Moffitt Cancer Center and Research Institute, Tampa, FL, USA \\ ${ }^{\mathrm{b}}$ University of California Davis Comprehensive Cancer Center, Sacramento, CA, USA
}

Received 1 March 2020

Accepted 15 April 2020

Pre-press 8 May 2020

Published 2 July 2020

\begin{abstract}
The treatment landscape of metastatic renal cell carcinoma (mRCC) is evolving very rapidly. Until recently, targeted monotherapy with vascular endothelial growth factor (VEGF)-tyrosine kinase inhibitors (TKIs) such as sunitinib, pazopanib and cabozantinib were considered the predominant frontline treatment options. In 2018, combination immune checkpoint inhibitor (ICI) therapy with ipilimumab and nivolumab was approved by the United States' Food and Drug Administration (FDA) for intermediate- and poor-risk patients. Subsequently, the FDA approved combination regimens consisting of a VEGFTKI with an immune checkpoint inhibitor for all risk categories: pembrolizumab-axitinib and avelumb-axitinib. In the context of these new developments and several ongoing trials in treatment naïve clear-cell $\mathrm{mRCC}$, there remains a dilemma among treating physicians about the choice of the most appropriate therapy as well as how to sequence these agents. In this review, we aim to highlight the available data on immunotherapy-based combinations and to provide a contemporary perspective on the optimal approach to patients with mRCC.
\end{abstract}

Keywords: Renal cell carcinoma, immunotherapy, first-line, systemic therapy, vascular endothelial growth factor (VEGF)

\section{INTRODUCTION}

Renal cell carcinoma (RCC) is among the top ten most common cancers in the world, accounting for approximately $90 \%$ of all adult renal malignancies [1]. It is estimated that there will be 73,750 new cases of RCC and an estimated 14,830 people will die of this disease in 2020 in the United States [2]. The treatment landscape of metastatic renalcell carcinoma (mRCC) has significantly changed over past several years. Previously, the standard first-line systemic treatment consisted of vascular endothelial growth factor (VEGF)-targeted tyrosine kinase inhibitor (TKI) monotherapy such as sunitinib, pazopanib or cabozantinib. The development

\footnotetext{
${ }^{*}$ Correspondence to: Rohit K Jain, MD, Department of Genitourinary Oncology, Moffitt Cancer Center, 12902 USF Magnolia Drive, Tampa, FL 33612, USA. E-mail: Rohit.jain@moffitt.org.
}

of T-cell checkpoint blockade with Programmed Cell Death protein 1 (PD-1) or cytotoxic T-lymphocyteassociated antigen 4 (CTLA-4) antibodies (Abs) has shown acceptable safety and durable anti-tumor activity in multiple malignancies, including RCC. Recently, multiple phase III randomized clinical trials comparing novel immune checkpoint inhibitor (ICI) combination therapies against sunitinib have shown improved efficacy outcomes, resulting in a major shift in frontline treatment strategies. This article will review these new therapeutic options available in the frontline space for $\mathrm{mRCC}$, focusing on the highest level of evidence presently available.

\section{DUAL CHECKPOINT INHIBITION}

RCC is considered to be among the most highly immunogenic tumors [3]. Cytotoxic T lympho- 
cyte antigen 4 (CTLA-4) is an immune checkpoint expressed on activated $T$ cells, and its interaction with B-7 family molecules results in an inhibitory signal that switches off $\mathrm{T}$ cell activity. The anti-CTLA-4 antibody, ipilimumab, prevents the deactivation of $\mathrm{T}$ cells by blocking this interaction [4]. Programmed death 1 (PD-1) is a protein expressed on activated T cells, and its binding to its ligands, PD-L1 or PD-L2 results in suppression of $\mathrm{T}$ cell activity [3]. Blockade of PD-1/PD-L1 axis by anti-PD-1 antibodies (nivolumab and pembrolizumab) or anti-PD-L1 antibodies (atezolizumab, avelumab, and durvalumab) allow $\mathrm{T}$ cells to maintain their anti-tumor activity in the tumor microenvironment. In the phase III CheckMate 025 study, nivolumab monotherapy was compared to everolimus in patients with mRCC progressed on prior VEGF-TKI therapy. There was significant improvement in overall survival (OS) with nivolumab 25 months (95\% CI 21.8 months- not estimable [NE]) vs 19.6 months (95\% CI 17.6-23.1 months) with everolimus, respectively [5]. Based on these results, nivolumab was approved as secondline treatment for this patient population. Motzer et al recently presented updated data of CheckMate 025 (with $>5$-years minimum follow-up), reporting that objective response rate (ORR) and OS remain superior with nivolumab. The ORR was $23 \%$ with nivolumab and $4 \%$ with everolimus, in addition, there were $28 \%$ ongoing responses seen with nivolumab compared to $18 \%$ with everolimus. The median duration of response was 18 months with nivolumab and 14 months with everolimus [6].

\section{Nivolumab plus Ipilimumab}

In advanced melanoma, the combination of ipilimumab with nivolumab showed substantial antitumor activity and had since been approved by the FDA [7, 8]. This combination was subsequently tested in the CheckMate 214 study where it was directly compared to single agent sunitinib [9]. In this trial, patients with clear-cell mRCC were randomized in $1: 1$ to receive either sunitinib $50 \mathrm{mg}$ on standard schedule or the combination of nivolumab $3 \mathrm{mg} / \mathrm{kg}$ plus ipilimumab $1 \mathrm{mg} / \mathrm{kg}$ (N3I1) intravenously every 3 weeks for 4 doses, followed by nivolumab $3 \mathrm{mg} / \mathrm{kg}$ monotherapy maintenance every 2 weeks. Based on prior phase I data, this combination was considered to have a tolerable safety profile. Treatment naive patients were included and stratified based on international mRCC database consortium (IMDC) criteria. The co-primary endpoints were ORR, PFS, and OS in the intermediate- and poor-risk groups. Secondary endpoints were ORR, PFS, and OS in the intention to treat (ITT) population and the assessment of adverse events. A total of 1096 patients were enrolled with 820 belonging to the intermediateand poor-risk groups. Five-hundred and fifty patients were randomized on the N3I1 arm and 546 on the sunitinib arm. Of these, 425 patients in the N3I1 arm and 422 in the sunitinib arm were in the intermediate- and poor-risk categories respectively. The ORR in the intermediate/poor-risk group was $42 \%(95 \%$ CI $37-47 \%)$ in the N3I1 arm and $27 \%$ (95\% CI $22-31 \%)(P<0.001)$ in the sunitinib arm, with complete responses (CR) in $9 \%$ and $1 \%$ patients respectively. The N3I1 combination showed a significant improvement in OS over sunitinib (hazard ratio [HR] $0.63,99.8 \%$ CI $0.44-0.89, P<0.001)$. The median OS was not reached for N3I1 arm (95\% CI 28.2 months-NE) versus 26 months on sunitinib arm (95\% CI 22.1 months-NE), at median followup of 25.2 months. The median duration of response (DOR) in the combination N3I1 arm was also not reached (95\% CI 21.8 months-NE) and was 18.2 months (95\% CI 14.8 months-NE) for sunitinib. The median PFS did not reach statistical significance with 11.6 months (95\% CI 8.7-15.5 months) in the N3I1 arm as compared to 8.4 months $(95 \%$ CI $7.0-10.8$ months) in the sunitinib arm (HR 0.82, $P=0.03$ ). Treatment-related adverse events (TRAEs) of any grade occurred in $93 \%$ of patients on the N3I1 arm and $97 \%$ on the sunitinib arm. Forty-six percent patients developed grade 3-4 adverse events (AEs) on N3I 1 arm and $63 \%$ on sunitinib arm, while $22 \%$ and $12 \%$ discontinued treatment, respectively. Thirtyfive percent patients on N3I1 arm were treated with high-dose corticosteroids due to immune-mediated adverse events including skin, endocrine, gastrointestinal, pulmonary, hepatic, and renal toxicities. Based on these results, the US FDA approved the combination of nivolumab and ipilimumab for the first-line treatment of intermediate- and high-risk clear-cell mRCC patients on April 16, 2018.

The updated results of Checkmate 214 trial with an extended median follow up of 32.4 months were recently published [10]. In this update, the ipilimumab plus nivolumab combination maintained superiority over sunitinib in intermediate- and poorrisk patients in the three co-primary efficacy endpoints. Importantly, the OS benefit was maintained with the combination in both the intermediate- and poor-risk and ITT populations. In this extended follow up period, PFS benefit was noted with the median 
PFS of 8.2 months (95\% CI 6.9-10 months) with the N3I1 arm as compared to 8.3 months (95\% CI 7-8.8 months) with the sunitinib arm (HR 0.77, 95\% CI $0.65-0.9, P=0.0014)$. Eleven percent patients developed durable CR with $88 \%$ patients yet with ongoing CR. In this analysis, the new and consistent definition of corticosteroids use to manage immune-mediated side effects was used and noted that only $29 \%$ of patients needed it compared to earlier data of $35 \%$.

Tannir et al recently presented the updated results of this trial with 42-month minimum follow up and 49-month median follow up [11]. In the primary intermediate-/poor-risk group and in the ITT population, the OS benefit was maintained with ipilimumab and nivolumab. The median OS was 47 months (95\% CI 35.6 months-NE) in the N3I1 arm as compared to 26.6 months (95\% CI 22.1-33.5 months) in the sunitinib arm (HR 0.66, 95\% CI 0.55-0.8, $P<0.0001)$. In the ITT population, the OS probabilities at 42 months were consistently higher with N3I1 (56\%) compared to sunitinib (47\%). Among the complete responders, $34 \%$ were still on treatment, $47 \%$ did not require subsequent treatment and the median DOR has not been reached. Among the complete responders $86 \%$ had ongoing response with only $14 \%$ patients relapsed. The median duration of treatmentfree survival among complete responders was 34.6 months.

It was noted under exploratory analyses that N3I1did not fare as well in favorable-risk patients. The ORR was $52 \%$ (95\% CI 43-61\%) with sunitinib versus $29 \%$ (95\% CI 21-38\%) with N3I1. The median PFS was 15.3 months (95\% CI 9.7-20.3 months) versus 25.1 months (95\% CI 20.9 monthsNE) (HR 2.18, 99.1\% CI 1.29-3.68, $P<0.001$ ), also favoring sunitinib. At 30-months follow-up, survival probability was slightly higher with sunitinib than the combination. With 42 months minimum followup, the difference in OS between treatment groups remained statistically insignificant (HR 1.19, 95\% CI $0.77-1.85, P=0.44)$. Median OS for either arm has not yet been reached. Thirteen percent patients with N3I1 achieved CR with $69 \%$ ongoing responses compared to $6 \%$ with sunitinib with $54 \%$ ongoing responses.

\section{VEGF-TARGETED THERAPY AND CHECKPOINT INHIBITION}

As a consequence of VHL gene disruption in RCC of the clear cell type, the downstream VEGF pathway plays a critical role in mRCC biology and clinical behavior. VEGF TKIs/VEGF blockers not only inhibit neo-angiogenesis, but also modulate the host tumor immune micro-environment (TIME), likely also contributing to anti-tumor activity [4]. VEGF overexpression leads to inhibition of dendritic cell maturation, increased myeloid-derived suppressor cells (MDSCs) and Treg (T regulatory) cells, and decreased cytotoxic $\mathrm{T}$ cell infiltration, thus suppressing both innate and adaptive immune responses [12-15]. In preclinical murine models, combination of anti-PD-1 and sunitinib resulted in increased tumor infiltrating lymphocytes, a reduction in MDSCs and reversal of immune response. Given that VEGF-directed therapies could potentially reverse the immunosuppressive effect in TIME, multiple trials in combination with ICIs were pursued.

\section{Nivolumab plus Sunitinib or Pazopanib}

Checkmate 016 was a multicenter, open-label phase I study that tested the combination of nivolumab with either sunitinb or pazopanib [16]. The starting dose of nivolumab was $2 \mathrm{mg} / \mathrm{kg}$ every 3 weeks, with planned escalation to $5 \mathrm{mg} / \mathrm{kg}$ every 3 weeks. This was combined with sunitinib at $50 \mathrm{mg} /$ day, 4 weeks on/2 weeks off or pazopanib $800 \mathrm{mg} /$ day until progression/unacceptable toxicity. Primary endpoints were safety and tolerability; secondary endpoints included PFS, DOR and PFS. One-hundred and ninety-four patients were enrolled; 33 patients received nivolumab plus sunitinib $(\mathrm{N}+\mathrm{S})$ while 20 received nivolumab plus pazopanib $(\mathrm{N}+\mathrm{P})$ combination. In the dose-escalation phase of the $\mathrm{N}+\mathrm{P}$ combination, there were signs of early hepatic toxicity; thus, this doublet did not proceed to the expansion phase. In $\mathrm{N}+\mathrm{S}$ arm, 7 patients completed nivolumab dose-escalation; the remaining 26 patients were then included in the dose-expansion phase with nivolumab $5 \mathrm{mg} / \mathrm{kg}$. In both the arms, $100 \%$ patients experienced TRAEs of any grade. About $81.8 \%$ patients experienced grade 3 and $70 \%$ experienced a grade 4 TRAE.

In $\mathrm{N}+\mathrm{S}$ arm, the most common grade 3 or 4 TRAEs included hypertension (18.2\%), increased alanine aminotransferase [ALT] (18.2\%), increased aspartate aminotransferase [AST] $(9.1 \%)$, diarrhea $(9.1 \%)$, and fatigue $(9.1 \%)$. Similarly, in the $\mathrm{N}+\mathrm{P}$ combination, grade 3 or 4 TRAEs included hypertension (10\%), increased ALT (20\%), increased AST (20\%), diarrhea $(20 \%)$, and fatigue (15\%). Due to immune-mediated side effects, $39.4 \%$ in $\mathrm{N}+\mathrm{S}$ 
and $60 \%$ in $\mathrm{N}+\mathrm{P}$ combination required systemic corticosteroids. With the $\mathrm{N}+\mathrm{S}$ combination, the confirmed ORR was $54.5 \%$ (95\% CI 36.4-71.9\%) with CR in 2 patients. The median DOR was 60.2 weeks (95\% CI 37.1 weeks - NR[not reached]); and median PFS was 12.7 months (95\% CI 11.016.7 months). In the $\mathrm{N}+\mathrm{P}$ treatment arm the confirmed ORR was $45.0 \%$ (95\% CI 23.1-68.5\%) with no CRs. Median DOR was 30.1 weeks (95\% CI 12.1-174.1 weeks); and median PFS was 7.2 months (95\% CI 2.8-11.1 months). Although, these combinations showed some clinical benefit, they were not developed further due to unacceptable toxicities. The majority of these toxicities are due to off-target effects of these multitargeted TKIs. Axitinib, a more selective VEGF-receptor inhibitor with a narrower spectrum of toxicity, was hypothesized to be a more appropriate partner to include in immunotherapy based regimens.

\section{Pembrolizumab plus Axitinib}

This combination was initially tested in a multicenter phase Ib study that included an initial dose-finding phase followed by an expansion phase [17]. Fiftytwo treatment naive $\mathrm{mRCC}$ patients were treated with pembrolizumab $2 \mathrm{mg} / \mathrm{kg}$ every 3 weeks plus axitinib at $5 \mathrm{mg}$ orally twice a day. The primary endpoint was assessment of dose limiting toxicity (DLT) in the first 6 weeks. Secondary endpoints included ORR, DOR, PFS, and OS, among others. Three of eleven patients treated in the dose-finding phase developed DLTs. The maximum tolerated dose (MTD) for the expansion phase was determined to be pembrolizumab $2 \mathrm{mg} / \mathrm{kg}$ every 3 weeks plus axitinib $5 \mathrm{mg}$ orally twice daily. Sixty-five percent of patients reported grade 3-4 TRAEs including hypertension $(23 \%)$, diarrhea (10\%), fatigue (10\%), and increased ALT (8\%). Grade 3 immune-mediated adverse effects were seen in $19 \%$ patients, including diarrhea $(29 \%)$, increased ALT (13\%), hypothyroidism (13\%), and fatigue $(12 \%)$. The ORR was an impressive $73 \%$, with $8 \%$ of patients achieving CR. The median PFS was 20.9 months (95\% CI 15.4 months-NE); median DOR was 18.6 months (95\% CI 15.1 months-NE). At the median follow-up of 20.4 months the median OS was not reached.

The results of this phase Ib study led to the pivotal phase III KEYNOTE 426 study, comparing the efficacy and safety of pembrolizumab plus axitinib $(\mathrm{P}+\mathrm{A})$ to the control arm of single agent sunitinib. A total of 861 patients were randomized to either pembrolizumab $200 \mathrm{mg}$ every 3 weeks plus axitinib $5 \mathrm{mg}$ BID versus sunitinib $50 \mathrm{mg}$ orally, 4 weeks on and 2 weeks off (standard schedule) [18]. The dual primary end points were OS and PFS across all IMDC risk groups. Secondary end points included ORR, DOR and safety. Patients were stratified by IMDC risk categories: $31 \%$ were favorable-, $56 \%$ intermediate- and $12.5 \%$ poor-risk. Four-hundred and twenty-nine patients in the $\mathrm{P}+\mathrm{A}$ arm and 425 patients in the sunitinib arm received at least one dose of the assigned treatment with the median follow-up being 12.8 months.

Significantly longer median PFS of 15.1 months was seen in the combination arm versus 11.1 months with sunitinib (HR $0.69,95 \%$ CI $0.57-0.84$, $P<0.001)$. Overall survival was also significantly longer, with $89.9 \%$ (95\% CI 86.4-92.4\%) patients in $\mathrm{P}+\mathrm{A}$ arm alive at 12 months compared with $78.3 \%$ (95\% CI 66.3-77\%) patients on sunitinib (HR 0.53, 95\% CI $0.38-0.74, P<0.0001)$. The median survival was not reached in either group. The ORR was higher in $\mathrm{P}+\mathrm{A}$ arm, $59.3 \%$ (95\% CI 54.5-63.9\%) versus $35.7 \%$ (95\% CI 31.1- 40.4\%) with sunitinib $(P<0.001)$; and $\mathrm{CR}$ rate was $5.8 \%$ in the $\mathrm{P}+\mathrm{A}$ arm. The median DOR was not reached in the $\mathrm{P}+\mathrm{A}$ group and was 15.2 months in the sunitinib group. The benefits of $\mathrm{P}+\mathrm{A}$ combination with respect to OS and PFS were observed in all IMDC risk and PD-L1 expression categories. Ninety-eight percent patients in the $P+\mathrm{A}$ arm and $99.5 \%$ in the sunitinib arm developed TRAEs. Of these $75.8 \%$ patients developed $\geq$ grade 3 TRAEs in the $P+\mathrm{A}$ arm and $70.6 \%$ of the patients in the sunitinib arm. Diarrhea and hypertension was noted to be the most common TRAEs in both groups. Grade 3 or more TRAEs in $>10 \%$ patients were hypertension and increased ALT levels in the $P+\mathrm{A}$ group and hypertension in the sunitinib group. Four of eleven $(0.9 \%)$ patients died due to TRAEs in $P+\mathrm{A}$ arm while $7 / 15(1.6 \%)$ patients died in sunitinib arm. Based on these results, pembrolizumab plus axitinib was approved by the FDA for the frontline treatment of mRCC patients regardless of IMDC risk group on April 19, 2019.

\section{Avelumab plus Axitinib}

JAVELIN Renal 100 was an open-label, multicenter, dose-finding, and dose-expansion, phase $\mathrm{Ib}$ study combining avelumab with axitinib for the treatment naive mRCC patients[19]. In the dose-finding phase, patients received axitinib $5 \mathrm{mg}$ orally twice a day followed by avelumab $10 \mathrm{mg} / \mathrm{kg}$ intravenously 
every 2 weeks. The primary endpoint was DLT assessment in the first 4 weeks of treatment. Secondary endpoints included safety assessment, ORR, disease control rate (DCR), DOR, PFS, and OS.

A total of 55 patients were enrolled, 6 in the dosefinding cohort, and 49 in the expansion cohort. The MTD was established at avelumab $10 \mathrm{mg} / \mathrm{kg}$ intravenously every 2 weeks plus axitinib $5 \mathrm{mg}$ orally twice a day. TRAEs were seen in $96 \%$ patients, with $58 \%$ developing grade $3-4$ adverse events. Fortytwo percent patients developed immune-mediated adverse events, with 9\% having grade 3-4 severity. These included $29 \%$ with hypertension, and $7 \%$ each with high levels of ALT, amylase, lipase, and palmar-plantar erythrodysaesthesia syndrome. There was one case of treatment- related grade 5 autoimmune myocarditis. In the dose-finding cohort ORR was confirmed in $100 \%$ of the patients and $58 \%$ in the expansion cohort. Duration of response, PFS, and OS could not be assessed at the median follow-up of 52.1 weeks. Among PD-L1 positive patients $(\geq 1 \%$ expression on tumor-associated immune cells), the ORR was $63 \%$ compared to $36 \%$ in $<1 \%$ expression.

This was then followed by JAVELIN Renal 101, a pivotal phase III study comparing the combination of avelumab $10 \mathrm{mg} / \mathrm{kg}$ every 2 weeks plus axitinib $5 \mathrm{mg}$ twice a day $(\mathrm{A}+\mathrm{A})$ versus single agent sunitinib $50 \mathrm{mg}$ on a 4-week on, 2-week off standard dosing schedule in treatment naïve mRCC patients [20]. The primary endpoint was either PFS or OS among PD-L1 positive patients ( $\geq 1 \%$ positive staining of immune cells within tumor area). The secondary endpoints included PFS and OS regardless of PD-L1 expression, ORR and safety. A total of 886 patients were randomized with 442 to the $\mathrm{A}+\mathrm{A}$ arm and 444 to the sunitinib arm. Sixty-three percent patients had PD-L1 positive tumors, of which 270 were in combination arm and 290 in sunitinib arm. Among patients with PD-L1 positive tumors, A + A had significantly longer median PFS of 13.8 months $(95 \%$ CI 11.1 months-NE) compared to 7.2 months $(95 \%$ CI 5.7-9.7 months) with sunitinib (HR 0.61, 95\% CI $0.47-0.79, P<0.001)$. The median PFS regardless of PD-L1 status was 13.8 months (95\% CI 11.1 months-NE) with A + A arm versus 8.4 months (95\% CI 6.9-11.1 months) with sunitinib (HR 0.69, 95\% CI $0.56-0.84, P<0.001)$. In the interim analysis, in PD-L1 positive patients, death from any cause was observed in $14.3 \%$ patients in $\mathrm{A}+\mathrm{A}$ arm and $16.9 \%$ in sunitinib arm (HR $0.78,95 \%$ CI $0.55-1.08, P=0.14$ ) during median follow up of 12 months and 11.5 months, respectively. The ORR in the PD-L1positive tumors was $55.2 \%$ (95\% CI 49-61.2\%) with A+ A and $25.5 \%$ (95\% CI 20.6-30.9\%) with sunitinib. Complete responses were reported in $4.4 \%$ in the $\mathrm{A}+\mathrm{A}$ arm and $2.1 \%$ in the sunitinib arm, respectively. Ninety-nine percent patients in A + A arm and 99.3\% in sunitinib arm developed TRAEs. Of these $71.2 \%$ patients developed $\geq$ grade 3 TRAEs in $\mathrm{A}+\mathrm{A}$ arm and $71.5 \%$ of the patients in the sunitinib arm.

The most common TRAEs included diarrhea and hypertension in both groups. Grade 3 or more TRAEs in $>10 \%$ patients was hypertension in both the groups. Immune-related adverse events were reported in $38.2 \%$ patients, with $9 \%$ patients developing $\geq$ grade 3 events in $\mathrm{A}+\mathrm{A}$ arm. Eleven percent patients received high dose corticosteroids due to these immune-related adverse events in A + A arm. Death due to treatment toxicity occurred in $0.7 \%$ patients in $\mathrm{A}+\mathrm{A}$ arm and $0.2 \%$ in sunitinib arm. Based on these results, the combination of avelumab plus axitinib was approved by the US FDA as frontline treatment for treatment naive mRCC patients on May 14, 2019.

\section{Atezolizumab plus Bevacizumab}

IMmotion 150 was a randomized phase II study comparing the efficacy of atezolizumab alone, atezolizumab plus bevacizumab versus standard-of-care sunitinib [21]. Atezolizumab 1,200 mg intravenously every 3 weeks was given as single agent or in combination with bevacizumab at $15 \mathrm{mg} / \mathrm{kg}$ intravenously every 3 weeks. Sunitinib was administered using the standard dose-schedule. The primary endpoint was PFS assessment according to the PD-L1 expression $(<1$ or $\geq 1 \%)$ on the tumor-infiltrating immune cells (TIC). A total of 305 patients were enrolled and randomized in 1:1:1 ratio. In the ITT population, the median PFS was 11.7 months $(95 \%$ CI 8.4-17.3 months) in the atezolizumab plus bevacizumab arm, 6.1 months (95\% CI 5.4-13.6 months) in the atezolizumab monotherapy arm, and 8.4 months (95\% CI 7.0-14.0 months) in the suntinib arm (HR 1.00, 95\% CI 0.60-1.45 and HR 1.19, 95\% CI 0.82-1.71 respectively). Among the patients with PD-L1positive TIC, the combination arm showed mPFS of 14.7 months (95\% CI 8.2-25.1 months), 5.5 months (95\% CI 3.0-13.9 months) with atezolizumab monotherapy versus 7.8 months $(95 \%$ CI $3.8-10.8$ months) with sunitinib (HR $0.64,95 \%$ CI $0.38-1.08$ and HR $1.03,95 \%$ CI $0.63-1.67$ respectively). The ORR was $32 \%$ with the combination, $29 \%$ with sunitinib, and $25 \%$ with the atezolizumab monotherapy. In the PD-L1 positive patients, the ORR was $46 \%$ 
with the combination, $27 \%$ with sunitinib, and $28 \%$ with atezolizumab monotherapy. Forty-percent grade 3-4 TRAEs were observed with the combination, $57 \%$ with suntinib, and $17 \%$ with atezolizumab alone.

IMmotion 151 trial was a phase III trial in treatment naïve $\mathrm{mRCC}$ patients whose tumors had clear-cell and/or sarcomatoid RCC histology. In this trial, the combination of atezolizumab plus bevacizumab was compared to the control arm of single-agent suntinib [22]. A total of 915 patients were randomized in a 1:1 ratio to receive atezolizumab $1,200 \mathrm{mg}$ intravenously, with bevacizumab $15 \mathrm{mg} / \mathrm{kg}$ intravenously every 3 weeks or sunitinib on a standard doseschedule. Patients were stratified based upon PD-L1 expression. The co-primary endpoints included PFS in PD-L1 positive patients and OS in ITT patients. The secondary endpoints in ITT population included PFS, ORR, and DOR. A total of 915 patients were included in ITT; with 362 being PD-L1 positive. In the PD-L1 positive group, the mPFS was significantly longer with the combination treatment at 11.2 months (95\% CI 8.9-15 months) compared to 7.7 months (95\% CI 6.8-9.7 months) with sunitinib (HR $0.74,95 \%$ CI $0.57-0.96, p=0.0217)$. Similary, in the ITT population, the combination arm had a mPFS of 11.2 months (95\% CI 9.6-13.3 months) versus 8.4 months (95\% CI 7.5-9.7 months) in the sunitinib arm (HR 0.83, 95\% CI 0.70-0.97, $p=0.0219$ ). In PD-L1 positive patients, the ORR was $43 \%$ with the combination versus $35 \%$ with sunitinib. In the overall population, the ORR was $37 \%$ for the combination versus $33 \%$ for sunitinib. In the second interim analysis at median follow up of 24 months, $43 \%$ patients in the combination arm and $42 \%$ patients in sunitinib arm had died (HR 0.93, 95\% CI 0.76-1.14, $P=0.475$ ). Forty percent patients developed Grade 3-4 TRAEs in the combination arm and 54\% in sunitinib arm. According to the investigator review, this study met its primary endpoint of PFS benefit in the PD-L1 positive patients. However, it was not significant on the central review in PD-L1 positive patients (HR 0.93, 95\% CI 0.71-1.04) as well in ITT population (HR $0.88,95 \%$ CI $0.74-1.04$ ). The combination of Atezolizumab-Bevacizumab has not been approved by the US FDA and is therefore still considered investigational as of this writing.

\section{DISCUSSION}

The US FDA approval of ipilumumab and nivolumab in combination for previously untreated intermediate- and poor-risk category $\mathrm{mRCC}$ patients brought a major shift in the frontline space which was previously dominated by VEGF-TKIs such as sunitinib or pazopanib monotherapy. Subsequently, phase III randomized clinical trials comparing PD-1/PD-L1 plus a VEGF inhibitor combination to a sunitinib control arm have been reported. Two of these trials led to the FDA approval of ICIs/TKI combinations: avelumab-axitinib and pembrolizumb-axitinib. To-date there remain many other frontline combination studies such as lenvatinib plus ICIs that have either recently completed accrual but have not yet matured, or are still open and accruing (Table 1).

With many new combinations, it has become more complex to understand which patients truly benefit from monotherapy versus combination treatments. There is paucity of immune monotherapy data in frontline setting and none of the phase III combination studies included them as comparator arm. KEYNOTE-427 cohort A, was a phase II, single-arm, study to evaluate efficacy and safety of pembrolizumab monotherapy in treatment-naïve, clear-cell RCC patients [23]. It showed an ORR of $33.6 \%$ (95\% CI $24.8-43.4 \%$ ). In favorable- and intermediate/poor-risk patients the ORR was $27.5 \%$ and $37.3 \%$, respectively. The median PFS was 6.9 months (95\% CI 5.1 months-NR), and $86 \%$ of responders had response lasting at least 3 months. Seventy-three percent patients developed TRAE, including fatigue $(23 \%)$, pruritius $(22 \%)$, diarrhea $(16 \%)$, rash (13\%), and arthralgia (12\%). Grade 3-5 TRAE were seen in $18 \%$ of patients. These results suggest that pembrolizumab monotherapy could be considered for certain frail patients who would be unable to tolerate doublet agents or for those whom TKIs are contraindicated.

With these new practice changing developments, the important questions are how these immunotherapy novel combinations compare to each other and which regimen should be used in treatment naïve $\mathrm{mRCC}$ patients. For patients in favorable-risk catergory as per IMDC risk stratification, CheckMate 214 data suggests improved RR and PFS with sunitinib over ipilimumab/nivolumab combination[9]. NCCN guidelines suggest that combination of ipilimumab/nivolumab should not be the preferred treatment, but could be used as a category $2 \mathrm{~A}$ recommended treatment option. The combination of pembrolizumab and axitinib showed overall improved outcomes compared to sunitinib in KEYNOTE 426 [18]. However, the subset analysis 
Table 1

Ongoing frontline trials for $\mathrm{mRCC}$ patients

\begin{tabular}{|c|c|c|c|c|c|}
\hline Trial & NCT number & $\begin{array}{l}\text { Treatment } \\
\text { combination }\end{array}$ & Phase & $\begin{array}{l}\text { Number of } \\
\text { patients }\end{array}$ & $\begin{array}{l}\text { Primary } \\
\text { endpoints }\end{array}$ \\
\hline$\overline{\text { CLEAR }}$ & 02811861 & $\begin{array}{l}\text { Lenvatinib plus } \\
\text { pembrolizumab or lenvatinib } \\
\text { plus everolimus vs sunitinib }\end{array}$ & 3 & 1069 & PFS \\
\hline CheckMate 9ER & 03141177 & Nivolumab Plus cabozantinib & 3 & 638 & PFS \\
\hline \multirow[t]{2}{*}{ COSMIC 313} & 03937219 & $\begin{array}{l}\text { Cabozantinib plus Nivolumab } \\
\text { and Ipilimumab Versus } \\
\text { Nivolumab and Ipilimumab }\end{array}$ & 3 & 676 & PFS \\
\hline & 03729245 & $\begin{array}{l}\text { NKTR-214 plus nivolumab } \\
\text { vs Investigator choice (either } \\
\text { sunitinb or cabozantinib } \\
\text { monotherapy) }\end{array}$ & 3 & 600 & ORR, OS \\
\hline PIVOT 02 & 02983045 & $\begin{array}{l}\text { NKTR-214 plus ipilimumab } \\
\text { and nivolumab }\end{array}$ & $1 / 2$ & 780 & ORR \\
\hline \multirow[t]{2}{*}{ MARIO-3 } & 03961698 & $\begin{array}{l}\text { IPI- } 549 \text { plus atezolizumab } \\
\text { and bevacizumab }\end{array}$ & 2 & 90 & $\mathrm{CR}$ rate \\
\hline & 03634540 & PT2977 plus cabozantinib & 2 & 118 & ORR \\
\hline
\end{tabular}

$\mathrm{PFS}=$ progression-free survival; $\mathrm{OS}=$ overall survival; $\mathrm{CR}=$ complete response; $\mathrm{ORR}=$ overall response rate.

in favorable-risk patients showed that the confidence interval for OS crossed unity (HR $0.81,95 \%$ CI $0.53-1.24)$. Thus, within this risk category where OS is estimated in years, the use of this combination treatment with its associated toxicities and costs should be carefully considered. Other alternatives would include VEGF-TKI monotherapy. Loo et al have also proposed a treatment algorithm with pembrolizumab/axitinib combination as a treatment choice and cabozanitinb as an alternative for patients with immunotherapy contraindications [24]. In intermediate- and poor-risk categories both ipilimumab/nivolumab and pembrolizumab/axitinib combination could be considered based on the patient profile. In certain clinical conditions such as extensive disease burden, organ compromise due to visceral crisis, the higher response rates of pembrolizumb/axitinib could be highly desirable [4]. On the other hand, patients with uncontrolled hypertension, hypertensive crisis would be a more suited for ipililumab/nivolumab combination. However, with the higher incidence of autoimmune adverse effects with ipilimumab/nivolumab, a multidisciplinary team consisting of an endocrinologist, gastroenterologist and pulmonologist, along with other specialists would be ideal. Cabozantinib as firstline treatment could also be used in intermediateand poor-risk patients especially in patients ineligible for immunotherapy due to autoimmune conditions or solid organ transplantation, as well as a highly selected subset with extensive bony metastatic disease [24].
While the discovery of these combination treatments have resulted in good anti-cancer efficacy, it is also important to understand the management of TRAEs. The principal adverse events associated with ICI and VEGF-TKI and their respective management strategies are very different. Therefore it is essential to educate oncology professionals about these strategies. Early and accurate recognization of the implicated agent is critical. Urgent treatment with steroids may be required for auto-immune breakthrough toxicity; alternatively, holding the drug for few days may be sufficient for TKI-related adverse events. For example combination treatments such as pembrolizumab/axitinib or avelumab/axitinib can cause diarrhea, which could be attributed to ICI-induced auto-immune colitis or TKI-induced mucosal toxicity. Considering that axitinib has short half-life and symptoms improve after holding or discontinuing the drug, it would be very reasonable to hold axitinib first in certain contexts. If diarrhea does improve after holding axitinib, then the attribution of that $\mathrm{AE}$ to the TKI is most likely to be correct. If diarrhea persists despite axitinib hold, then it would be appropriate to attribute the AE to ICI and thus to start high dose steroids and hold or discontinue ICI therapy (dependent on toxicity severity).

Another important question is the sequencing of agents in the subsequent line of therapies. Dudani et al used the IMDC dataset to compare outcomes for patients treated with ipilimumab and nivolumab and compared with any first-line ICI-VEGF combination therapies [25]. There was no statistical difference 
between first-line response rates, time to treatment failure, time to next treatment and OS. They also reported that as part of subsequent line of treatment, $88 \%$ of second-line therapies were VEGF based. The patients who received ipilimumab and nivolumab combination and subsequently received VEGF inhibitors had higher response rate versus those who received immunotherapy-VEGF combination. The effectiveness of rechallenge with immune monotherapy in second-line for patients who progressed on immunotherapy combination in the first line is unclear.

Lack of predictive and prognostic markers in the decision-making process is another major limitation in the management of mRCC. PD-L1 has some predictive biomarker value as seen with immune monotherapy or doublet combination due to its strong coorelation to Teff immune gene signature, however, its not applicable to VEGF-TKI plus ICI combination $[18,19,20,21]$. Also the lack of standardized asssessments by using different kits and methods to evaluate PD-L1 expression (tumor or immune cells) limits its use [26]. The signature profile developed in IMmotion 150 used relative expression levels of genes associated with angiogenesis, immune and myeloid inflammation markers [21]. Javelin RENAL 101 biomarker analysis included expression of PD-L1, CD-8, gene expression profiling, and mutations/polymorphisms [27]. They developed gene signature based on immune-related genes and noticed that it was associated with PFS benefit in the avelumb/axitinib arm. When IMmotion 150 gene signature was applied in Javelin Renal 101 there was no difference in PFS in the two arms [27]. Thus, at the present time there are no effective biomarkers that can be incorporated in treatment algorithm and future studies are warranted.

\section{CONCLUSION}

Currently, immunotherapy-based combination regimens are the appropriate frontline treatment for mRCC patients based on IMDC risk stratification. Among these options, there is no clearly superior strategy with respect to efficacy. The important thing to consider will be the side effect profile of these combinations and tailoring according to individual patient comorbidities and physician expertise. Other important factors contributing to decision making will include cost, convenience, logistics, prognostic categories, and patient preference. Results of recently completed or ongoing trials in the frontline setting are eagerly anticipated as they may again transform the therapeutic landscape of mRCC.

\section{ACKNOWLEDGMENTS}

The authors have no acknowledgments.

\section{FUNDING}

Dr. Lara is supported in part by National Cancer Institute Cancer Center Support Grant P30 CA093373.

\section{AUTHOR CONTRIBUTIONS}

Both the authors contributed equally in inception, interpretation of data and writing the manuscript.

\section{CONFLICT OF INTEREST}

RKJ: Honoraria: Dava Oncology; Advisory board: Pfizer; Speakers' Bureau: Astellas.

PLJ: Honoraria: Pfizer; Consulting or Advisory Role: Exelixis, Pfizer, AstraZeneca, Bayer AG, Genentech, Roche, Janssen Pharmaceuticals, Bristol-Myers Squibb, AbbVie, Turnstone Bio, Foundation Medicine, Merck, CellMax Life, Nektar; Research Funding: Millennium Pharmaceuticals (Inst), Polaris (Inst), GlaxoSmithKline (Inst), Genentech (Inst), Aragon Pharmaceuticals (Inst), Janssen Pharmaceuticals (Inst), Heat Biologics (Inst), TRACON Pharma (Inst), Merck (Inst), Pharmacyclics (Inst), Incyte (Inst).

\section{REFERENCES}

[1] American Cancer Society. Cancer Facts \& Figures 2019. Atlanta GACS.

[2] Siegel RL, Miller KD, Jemal A. Cancer statistics, 2020. CA Cancer J Clin. 2020;70(1):7-30. doi:10.3322/caac.21590

[3] Massari F, Santoni M, Ciccarese C, Santini D, Alfieri S, Martignoni G, et al. PD-1 blockade therapy in renal cell carcinoma: current studies and future promises. Cancer Treat Rev. 2015;41(2):114-21. doi:10.1016/j.ctrv.2014.12.013

[4] Amin A, Hammers H. The Evolving Landscape of Immunotherapy-Based Combinations for Frontline Treatment of Advanced Renal Cell Carcinoma. Front Immunol. 2018;9:3120. doi:10.3389/fimmu.2018.03120

[5] Motzer RJ, Escudier B, McDermott DF, George S, Hammers HJ, Srinivas S, et al. Nivolumab versus Everolimus in Advanced Renal-Cell Carcinoma. N Engl J Med. 2015;373(19):1803-13. doi:10.1056/NEJMoa1510665

[6] Motzer RJ, Tykodi SS, Escudier B, Oudard S, Hammers HJ, McDermott DF, et al. Final analysis of the CheckMate 
025 trial comparing nivolumab (NIVO) versus everolimus (EVE) with $>5$ years of follow-up in patients with advanced renal cell carcinoma (aRCC). J Clin Oncol. 2020;38(suppl 6; abstr 617). 2020.

[7] Wolchok JD, Kluger H, Callahan MK, Postow MA, Rizvi NA, Lesokhin AM, et al. Nivolumab plus ipilimumab in advanced melanoma. N Engl J Med. 2013;369(2):122-33. doi:10.1056/NEJMoa1302369

[8] Larkin J, Chiarion-Sileni V, Gonzalez R, Grob JJ, Cowey $\mathrm{CL}$, Lao CD, et al. Combined Nivolumab and Ipilimumab or Monotherapy in Untreated Melanoma. N Engl J Med. 2015;373(1):23-34. doi:10.1056/NEJMoa1504030

[9] Motzer RJ, Tannir NM, McDermott DF, Aren Frontera O, Melichar B, Choueiri TK, et al. Nivolumab plus Ipilimumab versus Sunitinib in Advanced RenalCell Carcinoma. N Engl J Med. 2018;378(14):1277-90. doi:10.1056/NEJMoa1712126

[10] Motzer RJ, Rini BI, McDermott DF, Aren Frontera O, Hammers HJ, Carducci MA, et al. Nivolumab plus ipilimumab versus sunitinib in first-line treatment for advanced renal cell carcinoma: extended follow-up of efficacy and safety results from a randomised, controlled, phase III trial. Lancet Oncol. 2019. doi:10.1016/S1470-2045(19)30413-9

[11] Tannir NM, McDermott DF, Escudier B, Hammers HH, Aren OR, Plimack ER, et al. Overall survival and independent review of response in CheckMate 214 with 42-month follow-up: First-line nivolumab+ipilimumab $(\mathrm{N}+\mathrm{I})$ versus sunitinib (S) in patients (pts) with advanced renal cell carcinoma (aRCC). J Clin Oncol. 2020;38(suppl 6; abstr 609). 2020.

[12] Gabrilovich DI, Chen HL, Girgis KR, Cunningham HT, Meny GM, Nadaf S, et al. Production of vascular endothelial growth factor by human tumors inhibits the functional maturation of dendritic cells. Nat Med. 1996;2(10):1096103.

[13] Gabrilovich DI, Nagaraj S. Myeloid-derived suppressor cells as regulators of the immune system. Nat Rev Immunol. 2009;9(3):162-74. i:10.1038/nri2506

[14] Maenhout SK, Thielemans K, Aerts JL. Location, location, location: functional and phenotypic heterogeneity between tumor-infiltrating and non-infiltrating myeloid-derived suppressor cells. Oncoimmunology. 2014;3(10):e956579. doi:10.4161/21624011.2014.956579

[15] Voron T, Colussi O, Marcheteau E, Pernot S, Nizard $\mathrm{M}$, Pointet AL, et al. VEGF-A modulates expression of inhibitory checkpoints on CD8+ T cells in tumors. J Exp Med. 2015;212(2):139-48. doi:10.1084/jem.20140559

[16] Amin A, Plimack ER, Ernstoff MS, Lewis LD, Bauer TM, McDermott DF, et al. Safety and efficacy of nivolumab in combination with sunitinib or pazopanib in advanced or metastatic renal cell carcinoma: the CheckMate 016 study. J Immunother Cancer. 2018;6(1):109. doi:10.1186/s40425018-0420-0

[17] Atkins MB, Plimack ER, Puzanov I, Fishman MN, McDermott DF, Cho DC,9 et al. Axitinib in combination with pembrolizumab in patients with advanced renal cell cancer: a non-randomised, open-label, dose-finding, and doseexpansion phase Ib trial. Lancet Oncol. 2018;19(3):405-15. doi:10.1016/S1470-2045(18)30081-0
[18] Rini BI, Plimack ER, Stus V, Gafanov R, Hawkins R, Nosov D, et al. Pembrolizumab plus Axitinib versus Sunitinib for Advanced Renal-Cell Carcinoma. N Engl J Med. 2019;380(12):1116-27. doi:10.1056/NEJMoa1816714

[19] Choueiri TK, Larkin J, Oya M, Thistlethwaite F, Martignoni M, Nathan P, et al. Preliminary results for avelumab plus axitinib as first-line therapy in patients with advanced clear-cell renal-cell carcinoma (JAVELIN Renal 100): an open-label, dose-finding and dose-expansion, phase Ib trial. Lancet Oncol. 2018;19(4):451-60. doi:10.1016/S14702045(18)30107-4

[20] Motzer RJ, Penkov K, Haanen J, Rini B, Albiges L, Campbell MT et al. Avelumab plus Axitinib versus Sunitinib for Advanced Renal-Cell Carcinoma. N Engl J Med. 2019;380(12):1103-15. doi:10.1056/NEJMoa1816047

[21] McDermott DF, Huseni MA, Atkins MB, Motzer RJ, Rini BI, Escudier B, et al. Clinical activity and molecular correlates of response to atezolizumab alone or in combination with bevacizumab versus sunitinib in renal cell carcinoma. Nat Med. 2018;24(6):749-57. doi:10.1038/s41591-0180053-3

[22] Rini BI, Powles T, Atkins MB, Escudier B, McDermott DF, Suarez C et al. Atezolizumab plus bevacizumab versus sunitinib in patients with previously untreated metastatic renal cell carcinoma (IMmotion151): a multicentre, open-label, phase III, randomised controlled trial. Lancet. 2019;393(10189):2404-15. doi:10.1016/S01406736(19)30723-8

[23] Donskov F, McDermott DF, Lee JL, Szczylik C, Malik J, Alekseev BY, et al. KEYNOTE-427 COHORT A: Pembrolizumab monotherapy as first-line therapy in advanced clear cell renal cell carcinoma (CCRCC). Annals of Oncology (2018) 29 (suppl_8): viii303-viii331 101093/annonc/mdy283. 2018.

[24] Loo V, Salgia M, Bergerot P, Philip EJ, Pal SK. FirstLine Systemic Therapy for Metastatic Clear-Cell Renal Cell Carcinoma: Critical Appraisal of Emerging Options. Target Oncol. 2019;14(6):639-45. doi:10.1007/s11523019-00676-y

[25] Dudani S, Graham J, Wells JC, Bakouny Z, Pal SK, Dizman N, et al. First-line Immuno-Oncology Combination Therapies in Metastatic Renal-cell Carcinoma: Results from the International Metastatic Renal-cell Carcinoma Database Consortium. Eur Urol. 2019. doi:10.1016/j.eururo.2019.07.048

[26] Zhu J, Armstrong AJ, Friedlander TW, Kim W, Pal SK, George DJ et al. Biomarkers of immunotherapy in urothelial and renal cell carcinoma: PD-L1, tumor mutational burden, and beyond. J Immunother Cancer. 2018;6(1):4. doi:10.1186/s40425-018-0314-1

[27] Choueiri TK, Albiges L, Haanen JBAG, Larkin J, Uemura M, Pal SK, et al. Biomarker analyses from JAVELIN Renal 101: Avelumab+axitinib ( $A+A x)$ versus sunitinib (S) in advanced renal cell carcinoma (aRCC). J Clin Oncol 2019; 37(Suppl 15): 101. 2019. 